\title{
La cueillette, la production et la commercialisation du miel dans le Département de Katiola (Centre-Nord, Côte d'Ivoire)
}

\author{
Dido Fabrice KOUASSI ${ }^{1 *}$, Djakalia OUATTARA ${ }^{12}$, Siendou COULIBALY ${ }^{3}$ et \\ Kouakou Edouard N'GUESSAN ${ }^{1}$
}

\author{
${ }^{1}$ Laboratoire de Botanique, Université Félix Houphouët-Boigny, 22 BP 582 Abidjan 22, Côte d'Ivoire. \\ ${ }^{2}$ Centre National de Floristique (CNF), Université Félix Houphouët-Boigny, 22 BP 582 Abidjan 22, \\ Côte d'Ivoire. \\ ${ }^{3}$ UFR Agroforesterie, Université Jean Lorougnon Guédé, BP 150 Daloa, Côte d'Ivoire. \\ *Auteur correspondant ; E-mail : didofabricek@gmail.com, Tél : 0022508585868
}

\section{RESUME}

Récolté autrefois pour l'alimentation, du fait notamment de sa grande valeur nutritionnelle, le miel est devenu aujourd'hui un produit générateur de revenus substantiels pour les populations, malgré le caractère informel de ce secteur. Afin de mieux le comprendre et contribuer à sa valorisation, des investigations ont été menées sur la cueillette, la production et la commercialisation du miel dans le Département de Katiola (Côte d'Ivoire), durant l'année 2017. Ainsi, 87 personnes du secteur ont été interviewées, dont 11 apiculteurs modernes, 45 cueilleurs de miel ou "chasseurs de miel" et 31 commerçantes du miel. Il ressort que les apiculteurs modernes sont rares dans la zone de l'étude. La quasi-totalité du miel provient de la "chasse". Malheureusement, dans cette région savanicole, la "chasse de miel" entraîne l'abattage de plus de 100 arbres/an, ainsi que la destruction par le feu de centaines de colonies d'abeilles. Au plan économique, les apiculteurs modernes gagnent en moyenne 200000 francs CFA/an à travers la vente du miel, contre 45000 francs CFA/an pour les commerçantes et 10000 francs CFA/an pour les "chasseurs de miel". Un meilleur encadrement des activités apicoles pourrait permettre de promouvoir ce secteur.

(C) 2018 International Formulae Group. All rights reserved.

Mots clés: Apis mellifera, chasseur de miel, apiculture, commerce.

\section{Gathering, production and marketing of honey in Katiola Division (North-Central, Côte d'Ivoire)}

\begin{abstract}
Harvested in the past for food, especially because of its high nutritional value, honey has now become a product generating substantial income for the population, despite the informal nature of this sector. In order to better understand it and contribute to its valorization, investigations were carried out on the collection,
\end{abstract}


production and marketing of honey in the Department of Katiola (Ivory Coast), during the year 2017. Thus, 87 people from sector were interviewed, including 11 modern beekeepers, 45 honey gatherers or "honey hunters" and 31 honey traders. It appears that modern beekeepers are rare in the study area. Almost all of the honey comes from "hunting". Unfortunately, in this savannah region, the "hunt for honey" involves the slaughter of more than 100 trees / year, as well as the destruction by fire of hundreds of bee colonies. At the economic level, modern beekeepers earn on average 200,000 CFA francs / year through the sale of honey, against 45,000 CFA francs / year for shopkeepers and 10,000 CFA / year for "honey hunters". Better supervision of beekeeping activities could help promote this sector.

(C) 2018 International Formulae Group. All rights reserved.

Keywords: Apis mellifera, hunter of honey, beekeeping, trade.

\section{INTRODUCTION}

Le miel est la substance sucrée naturelle produite par l'abeille Apis mellifera à partir du nectar de plantes et de miellat, qu'elle butine, transforme, entrepose et laisse mûrir dans les rayons de la ruche (UE, 2014). Il constitue un produit de grand intérêt pour l'être humain qui l'exploite depuis plus de 4500 ans pour des besoins alimentaires, thérapeutiques et économiques (FAO, 2010). En Côte d'Ivoire, plusieurs personnes sont engagées dans la cueillette, la production et la commercialisation du miel. La cueillette du miel ou "chasse au miel" consiste à repérer pendant la journée, des colonies sauvages d'abeilles dans des trous d'arbres ou entre des rochers, et à récolter le miel la nuit après destruction de la colonie par le feu (Yédomonhan et Akoègninou, 2009). A l'instar de plusieurs régions de la zone de savanes, au Nord de la Côte d'Ivoire, le Département de Katiola est une zone de référence pour la production de miel. En effet, l'initiation des populations ivoiriennes à l'apiculture moderne a commencé par ce département (Hussien, 2001). Un projet apicole de la Société pour le Développement des Productions Animales (SODEPRA), en coopération avec l'agence Allemande de la coopération technique (GTZ), a été initié dans cette partie de la Côte d'Ivoire au début des années 80 , afin de valoriser les produits de la ruche (miel, cire, pollen, etc.). Ainsi, la production, la "chasse" et le commerce du miel sont autant d'activités menées par les populations du Département de Katiola. Des revenus substantiels sont ainsi générés chaque année. Malgré cela, peu de données scientifiques se rapportant à ces activités à impact non moins négligeable sur la biodiversité, sont disponibles à ce jour. Le présent travail vise donc à fournir des informations d'actualité sur les activités autour du miel dans cette région de la Côte d'Ivoire, afin de mieux orienter les actions de gestion et de développement à y mener.

\section{MATERIEL ET METHODES}

\section{Zone de l'étude}

Le Département de Katiola est situé dans la Région du Hambol au Centre-Nord de la Côte d'Ivoire, entre $8^{\circ} 46^{\prime} 70.02^{\prime \prime}$ et $8^{\circ} 87^{\prime}$ $23.97 "$ de latitudes Nord et $5^{\circ} 63^{\prime} 31.18^{\prime \prime}$ et $4^{\circ}$ 75' 95.62" de longitudes Ouest (Figure 1). La population est dominée par les autochtones Tagbanan et Mangôrô. A ceux-ci s'ajoutent plusieurs groupes allochtones (maliens, burkinabés, etc.) et allogènes (Baoulé, Senoufo, etc.). Selon le Recensement Général de la Population et de l'Habitat (RGPH) de 2014, la population est estimée à 106905 habitants. Le climat du département est de type tropical sub-humide (Coulibaly, 2009). La pluviométrie annuelle oscille entre 1100 $\mathrm{mm}$ et $1200 \mathrm{~mm}$, avec une température 
moyenne d'environ $27^{\circ} \mathrm{C}$ (Kouakou et al., 2010). La végétation, constituée de savanes et de forêts semi-décidues (Koulibaly et al., 2006), est caractéristique du secteur subsoudanais du domaine soudanais. Les sols sont de types ferralitiques à texture argilosableuse et gravillonnaire (Coulibaly, 2009).

Deux raisons majeures ont motivé le choix du Département de Katiola. Dans un premier temps, c'est une zone de référence en matière d'apiculture, car l'initiation à la pratique apicole moderne en Côte d'Ivoire a commencé par cette région (Hussien, 2001). Dans un second temps, on y trouve des apiculteurs organisés en coopérative reconnue par l'autorité.

\section{Collecte des données}

Sept (07) localités (Katiola, Fronan, Kationon, Timbé, Foro-Foro, Touro et Nikolo) ont été choisies de manière aléatoire, en tenant compte de la diversité des trois souspréfectures du Département (Katiola, Fronan et Timbé).

Les données ont été collectées à travers une enquête basée sur un questionnaire semistructuré. Des interviews directes ont ciblé trois groupes d'informateurs intervenant dans le domaine apicole, à savoir, les apiculteurs modernes, les "chasseurs de miel" et les commerçantes. Le questionnaire a été soumis individuellement ou sous forme de focus group. Cette dernière méthode permet d'enrichir les informations recueillies lors de l'interview individuel (Lebel et al., 2002). Des discussions semi-dirigées, faisant usage d'un check-list de questions ouvertes flexibles où de nouvelles questions ou pistes d'interrogations émergent tout au long de l'entretien (Tamboura et al., 1998), ont été réalisées. Ainsi, de nombreuses informations se rapportant à la production, la cueillette et la commercialisation du miel pour les années 2016 et 2017 ont été recueillies. Cependant, nous n'avons pu recueillir que le coût estimatif de de l'ensemble de l'équipement apicole pour l'année 2017, car certains apiculteurs ont acquis leur matériel depuis plus de 30 ans (avant la dévaluation du franc CFA), alors que d'autres l'on reçu pour un franc symbolique de la part de donateurs (structure de formation, projet).

\section{Analyse des données}

Les données collectées ont été encodées pour une analyse descriptive avec le logiciel Microsoft Office Excel 2007. Pour l'évaluation de la rentabilité du commerce du miel, la méthode coûts/recettes a été utilisée (Dieye et al., 2002 ; Tousso 2005). Les coûts ou dépenses totaux de production se rapportent au matériel et à diverses dépenses. Les recettes brutes ont été estimées à partir des flux monétaires issus de la vente du miel. Concernant la "chasse au miel", le bénéfice de l'activité a été calculé de manière indépendante pour chaque informateur, selon qu'il appartient ou non à un groupement. Le bénéfice moyen par chasseur a ainsi été estimé.

Le logiciel XLStat 2014 a servi à réaliser les tests de corrélations de Pearson et de Kruskal-Wallis. Ce dernier a été effectué en lieu et place du test d'ANOVA lorsque l'absence de normalité était constatée à travers le test de Shapiro-Wilk. Le niveau de significativité choisi pour ces tests de comparaison de moyennes était de 5\%. Chaque fois que la probabilité calculée était significative $(\mathrm{p}<5 \%)$, le test de Dunn a été effectué afin de comparer deux à deux les moyennes et d'apprécier la significativité de leur différence. 


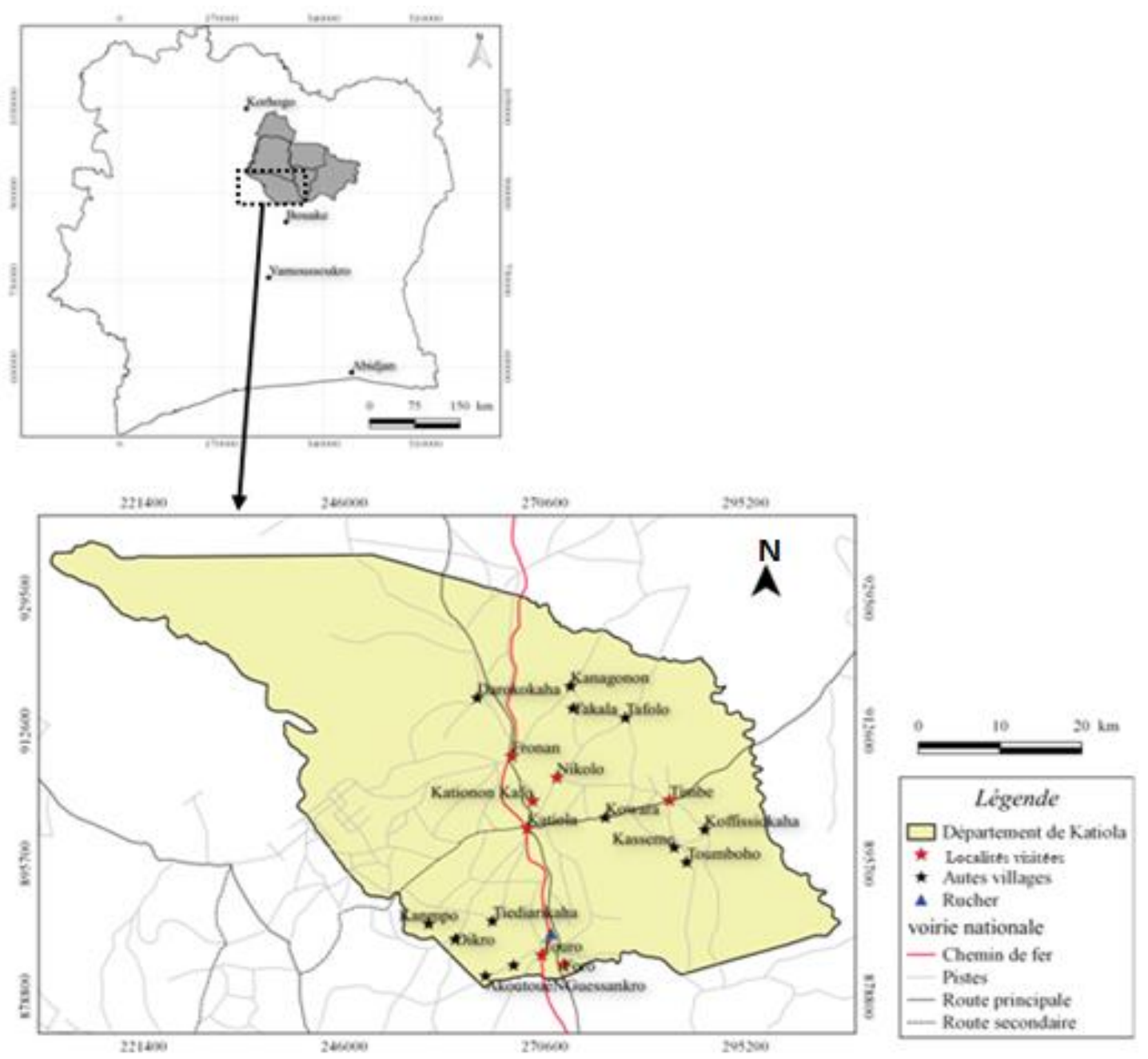

Figure 1 : Carte du Département de Katiola au Centre-Nord de la Côte d'Ivoire.

\section{RESULTATS}

\section{Profil des informateurs}

$\mathrm{Au}$ total, 87 personnes ont été interviewées, dont $12,64 \%$ d'apiculteurs modernes, $51,73 \%$ de "chasseurs de miel" et $35,63 \%$ de commerçantes de miel. Leur âge varie de 15 à 71 ans, avec une moyenne de 35 ans. La classe d'âges [36-45 ans] est prédominante, avec $37 \%$, tandis que la classe [46 ans et plus] comporte le plus faible taux qui est de $12 \%$ (Figure 2). Les apiculteurs modernes ont un âge compris entre 35 et 71 ans, avec une moyenne de 48 ans. Les "chasseurs de miel" sont âgés de 15 à 57 ans, avec une moyenne de 31 ans. Enfin, l'âge des commerçantes varie de 21 à 67 ans, avec une moyenne de 36 ans.
Concernant le niveau d'instruction, $41 \%$ des informateurs sont analphabètes tandis que $29 \%$ ont atteint le secondaire (Tableau 1). Les plus forts taux de niveau d'instruction sont de $38 \%$ et $55 \%$ d'analphabètes respectivement chez les "chasseurs" et les commerçantes de miel, et de $64 \%$ de niveau secondaire chez les apiculteurs modernes.

Concernant le sexe, $64,37 \%$ des personnes enquêtées sont des hommes, dont $12,64 \%$ d'apiculteurs modernes et $51,73 \%$ de "chasseurs de miel". Chez ces deux derniers groupes acteurs, cette activité est secondaire. Par contre, les commerçantes de miel exercent le commerce comme activité principale, en 
associant au miel divers autres produits tels que les fruits, les légumes, le charbon de bois.

Parmi les personnes interviewées, 67\% sont des autochtones (Tagbanan), 27\% des allochtones (Senoufo, Baoulé, Malinké, etc.) et $6 \%$ des allogènes (Burkinabé et Malien).

\section{Pratique de la chasse au miel}

Sur les 45 "chasseurs de miel" interviewés, $18 \%$ chassent de manière solitaire et $82 \%$ en groupes de 2 à 6 personnes. Par ailleurs, $52 \%$ ont une expérience de 1 à 10 ans dans cette activité, $44 \%$ ont une expérience de 11 à 20 ans et $4 \%$ pratiquent cette activité depuis 21 ans au moins.

Les périodes de récolte du miel varient d'un chasseur à l'autre et selon les groupes. Ainsi, $47 \%$ des chasseurs pratiquent la chasse au miel de janvier à mars, 44\% la pratiquent de février à mars, $5 \%$ de février à avril, $2 \%$ s'y adonnent seulement en avril et $2 \%$ de janvier à avril.

Pour l'année 2016, chaque "chasseur" ou groupe de "chasseurs de miel" a récolté en moyenne 23,7 litres de miel (Tableau 2). Pour cela, il a exploré en moyenne 4 arbres dont 3 ont été abattus. En 2017, ce sont en moyenne 17,4 litres de miel qui ont été récoltés dans 3 arbres dont 2 ont été abattus. Le test statistique (Kruskal-Wallis) effectué, a montré qu'il n'y a pas de différences significative entre la quantité de miel récolté en 2016 et celle récoltée en $2017\left(x^{2}=1,67, \mathrm{p}=0,195\right)$. Aussi, pour chacune des deux années, il existe une corrélation linéaire hautement positive ( $\mathrm{p}$ $<0,0001$ ) entre le nombre d'arbre abattu pendant les récoltes et la quantité de miel récolté. Le coefficient de corrélation de Pearson est de 0,87 pour 2016 et 2017 (Figure $3)$.

Le prix de vente du miel issu de la chasse varie de 1000 à 2250 francs CFA/litre selon le lieu de vente, avec un prix moyen de 1610 francs CFA/litre. Ce miel est vendu sur le marché local du Département à divers types de clients. En effet, $64,45 \%$ de "chasseurs" ou groupes de "chasseurs de miel" vendent le fruit de leur activité uniquement aux commerçantes ; $31,11 \%$ le vendent à la fois à des particuliers et aux commerçantes. Seulement $4,44 \%$ vendent le miel uniquement à des particuliers.
La "chasse au miel" est une activité qui ne nécessite pratiquement aucun fond d'investissement. L'argent issu de la vente du miel constitue donc un bénéfice net pour les concernés. Ainsi, pour l'année 2016, au total 1067 litres de miel issus de la "chasse" ont été récoltés par l'ensemble des "chasseurs" enquêtés, pour un gain de 1684700 francs CFA. En 2017, un total de 783 litres de miel a été récolté, pour un bénéfice de 1209450 francs CFA.

La "chasse au miel" est une activité qui comporte, aux dires des chasseurs, quelques difficultés. En effet, $64,45 \%$ des chasseurs interviewés jugent l'activité difficile du fait de la rareté des colonies sauvages d'abeilles durant ces dernières années, 35,55\% d'entre eux la jugent difficile aussi bien pour la rareté des colonies que pour les piqûres d'abeilles lors de la récolte.

\section{Pratique de l'apiculture moderne}

Le Département de Katiola regorge de peu d'apiculteurs modernes. Toutefois, onze (11) apiculteurs ont pu être interviewés. Ils ont une ancienneté dans la pratique apicole variant de 2 à 50 ans, avec une moyenne de 15 ans. Plus de la moitié $(54,54 \%)$ de ces apiculteurs ont appris l'apiculture moderne auprès d'un ami, $27,27 \%$ ont bénéficié d'un programme de formation financé par la Banque Africaine de Développement (BAD) en 1994 et, enfin 18,18\% ont été formés par des Organisations Non Gouvernementales (ONG).

Au total, 172 ruches modernes ont été recensées pour 11 apiculteurs. Celles de type Langstroth représentent $76,16 \%$, contre $23,84 \%$ pour les ruches Kenyannes. Le nombre de ruchers détenus par un apiculteur varie de 1 à 4, chacun comprenant en moyenne 15 ruches. Pour les deux années 2016 et 2017, la production brute moyenne de miel pour un apiculteur a varié de 120,82 à 109,27 litres, soit de 9,22 à 8,92 litres par ruche (Tableau 3). Le test statistique (KruskalWallis) effectué montre qu'il n'y a pas de différence significative entre la quantité de miel récolté en 2016 et celle récoltée en 2017 $\left(x^{2}=0,13 \mathrm{p}=0,72\right)$. Pour ces deux années, le litre de miel a été vendu à 1955 francs CFA en moyenne. Ce qui correspond à une recette brute de 235909 francs CFA par apiculteur 
pour l'année 2016 et de 209727 francs CFA par apiculteur pour 2017.

Le coût moyen estimatif de tout l'équipement utilisé par un apiculteur (ruches, brosses, combinaisons, enfumoirs, récipients, etc.) pour la pratique de son activité est de 154545 francs CFA.

Vingt et sept pour cent $(27 \%)$ des apiculteurs vendent toute leur production via la Coopérative Ivoire Miel de Katiola (CIMKA). 18\% livrent une partie de leur miel à la coopérative, tandis que l'autre partie est vendue à des acheteurs venant des villes d'Abidjan ou de Bouaké. 55\% des apiculteurs vendent la totalité de leur production également à ces acheteurs.

S'agissant du site d'installation du rucher, tous les apiculteurs jugent propice un milieu ombrageux. Quant à la période de piégeage des essaims, $82 \%$ des apiculteurs estiment que la saison sèche est plus propice, quand $18 \%$ pensent que cet exercice peut se faire à toutes les périodes de l'année.

En ce qui concerne la récolte du miel, elle a lieu durant les cinq (5) premiers mois de l'année (de janvier à mai). Néanmoins, $82 \%$ des apiculteurs procèdent à la récolte de mars à mai, tandis que $18 \%$ préfèrent la période de janvier à mars.

L'apiculture moderne connaît certaines difficultés dont la plus courante est la désertion des ruches par les abeilles. En effet, tous les apiculteurs font face à ce problème fréquent, surtout après la récolte.

$\mathrm{La}$ comparaison de l'apiculture moderne à la "chasse au miel" a révélé une différence significative entre les quantités de miel récoltées, l'apiculture étant plus productive (Tableau 4).

\section{Commerce du miel}

Dans le Département de Katiola, le miel est essentiellement vendu par des femmes qui occupent les abords des voies principales et les marchés. Nous avons interviewé 31 femmes durant cette étude. Elles exercent cette activité depuis 2 à 30 ans, avec une moyenne de 13 ans. Elles s'approvisionnent toutes auprès des "chasseurs de miel". Le miel vendu par ces commerçantes provient essentiellement de la même localité où elles exercent leur activité (Figure 4). Toutefois, le miel vendu par celles exerçant dans la ville de Katiola est de diverses origines, avec une prédominance du miel issu de Tafiré et de Niakaramadougou.

Par ailleurs, chacune des commerçantes de miel s'approvisionne auprès de 2 à 10 fournisseurs ("chasseurs de miel"), avec une moyenne de 4 fournisseurs. Elles achètent le miel durant les 6 premiers mois de l'année (janvier-juin), principalement de janvier à mars (Figure 5).

La quantité moyenne de miel achetée par chaque commerçante était de 81,77 litres en 2016 et de 66,97 litres en 2017 (Tableau 5). Elles ont payé entre 1100 et 2000 francs CFA pour un litre de miel, soit 1500 francs CFA en moyenne. Le litre a été revendu entre 2000 et 2500 francs CFA, soit en moyenne à 2010 francs CFA.

En dehors du capital engagé pour l'achat du miel, les commerçantes font des dépenses supplémentaires liées au transport et à l'acquisition d'emballages (bouteilles de récupération en général) pour la vente au détail. Ainsi, en 2016, en moyenne 119630 francs CFA ont été dépensés par chaque commerçante pour l'achat du miel et 4890 francs CFA pour le transport et l'acquisition de bouteilles. En 2017, elles ont dépensé chacune 98240 francs CFA en moyenne pour l'achat du miel et 4130 francs CFA en moyenne pour le transport et les emballages. En définitive, le bénéfice net moyen obtenu par chaque commerçante a été de 56050 francs CFA en 2016 et de 46400 francs CFA en 2017.

En ce qui concerne le lieu de commercialisation, la quasi-totalité des commerçantes (90\%) vendent le miel localement (marché de leur lieu de résidence). Seules $10 \%$ d'entre elles le vendent également à Abidjan et à Bouaké, deux plus grandes villes du pays. Dans $78 \%$ des cas, elles le vendent uniquement au détail, donc directement aux consommateurs, $19 \%$ des cas à la fois au détail et en gros. Seules 3\% d'entre elles le vendent uniquement en gros.

Par ailleurs, deux difficultés majeures dans l'exercice de cette activité ont été soulignées. Il s'agit de la vente du miel qui se fait avec une certaine lenteur, reconnue par toutes les commerçantes et de tracasseries routières perpétrées par des agents des Eaux et Forêts, subies par 35,48\% d'elles. 


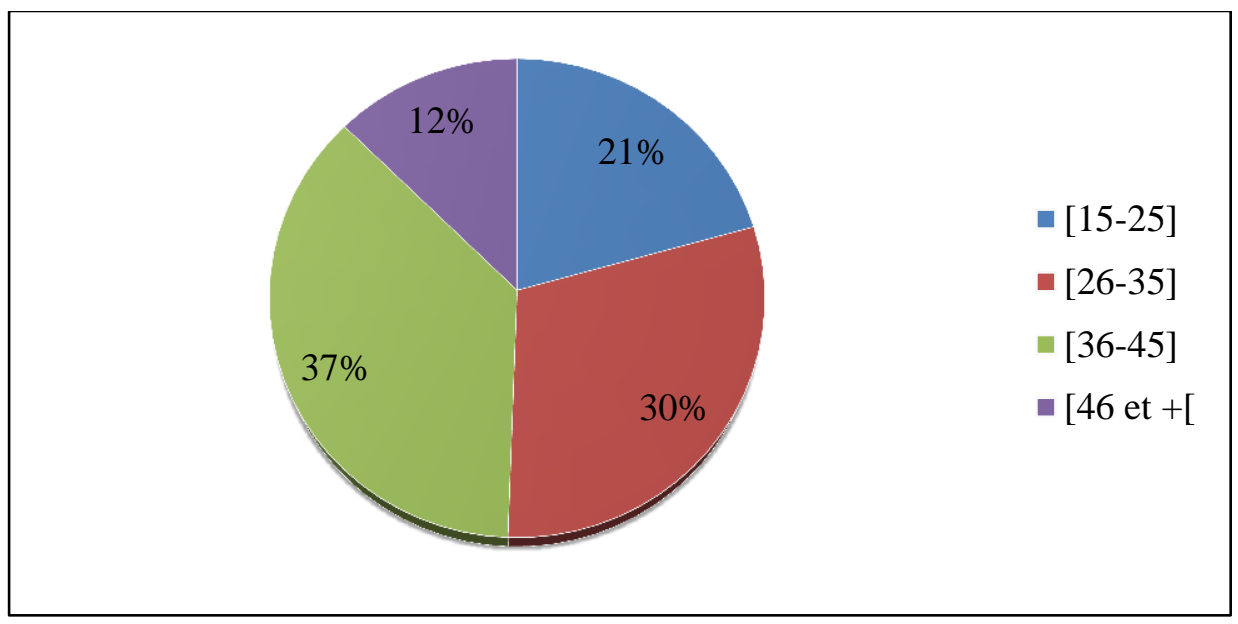

Figure 2 : Spectre des personnes interviewées selon la classe d'âges.

Tableau 1 : Niveau d'instruction des personnes interviewées.

\begin{tabular}{lccc}
\hline \multirow{2}{*}{ Acteur } & \multicolumn{3}{c}{ Niveau d'instruction } \\
\cline { 2 - 4 } & Analphabète (\%) & Primaire (\%) & Secondaire (\%) \\
\hline Apiculteurs & 18 & 18 & 64 \\
Chasseurs de miel & 38 & 31 & 31 \\
Commerçantes & 55 & 32 & 13 \\
Ensemble des acteurs & 41 & 30 & 29 \\
\hline
\end{tabular}

Tableau 2 : Données sur la chasse au miel en 2016 et 2017 dans le Département de Katiola.

\begin{tabular}{lll}
\hline Paramètre évalué & $\mathbf{2 0 1 6}$ & $\mathbf{2 0 1 7}$ \\
\hline Récolte totale de miel (litres) & 1067 & 783 \\
Nombre d'arbres explorés & 187 & 141 \\
Nombre d'arbres abattus & 132 & 101 \\
Récolte moyenne de miel par arbre exploré (litres) & $5,92 \pm 7,4$ & $5,8 \pm 7,96$ \\
Nombre d'équipes de chasseurs de miel & 37 & 37 \\
Nombre de chasseurs solitaires & 8 & 8 \\
Nombre total de chasseurs de miel & 114 & 113 \\
Récolte moyenne de miel par chasseur (litres) & $23,7 \pm 29,6^{\text {a }}$ & $17,4 \pm 23,3^{\text {a }}$ \\
Prix moyen de vente du litre de miel (F CFA) & $1610 \pm 301$ & $1610 \pm 301$ \\
Recette brute moyenne (F CFA) & $1684700 \pm 44935$ & $1209450 \pm 31890$ \\
Bénéfice moyen par chasseur (F CFA) & $12509 \pm 9690$ & $9747 \pm 7035$ \\
\hline Les valeurs moyennes affectées de la même lettre ne sont pas significativement différentes au seuil de 5\%.
\end{tabular}




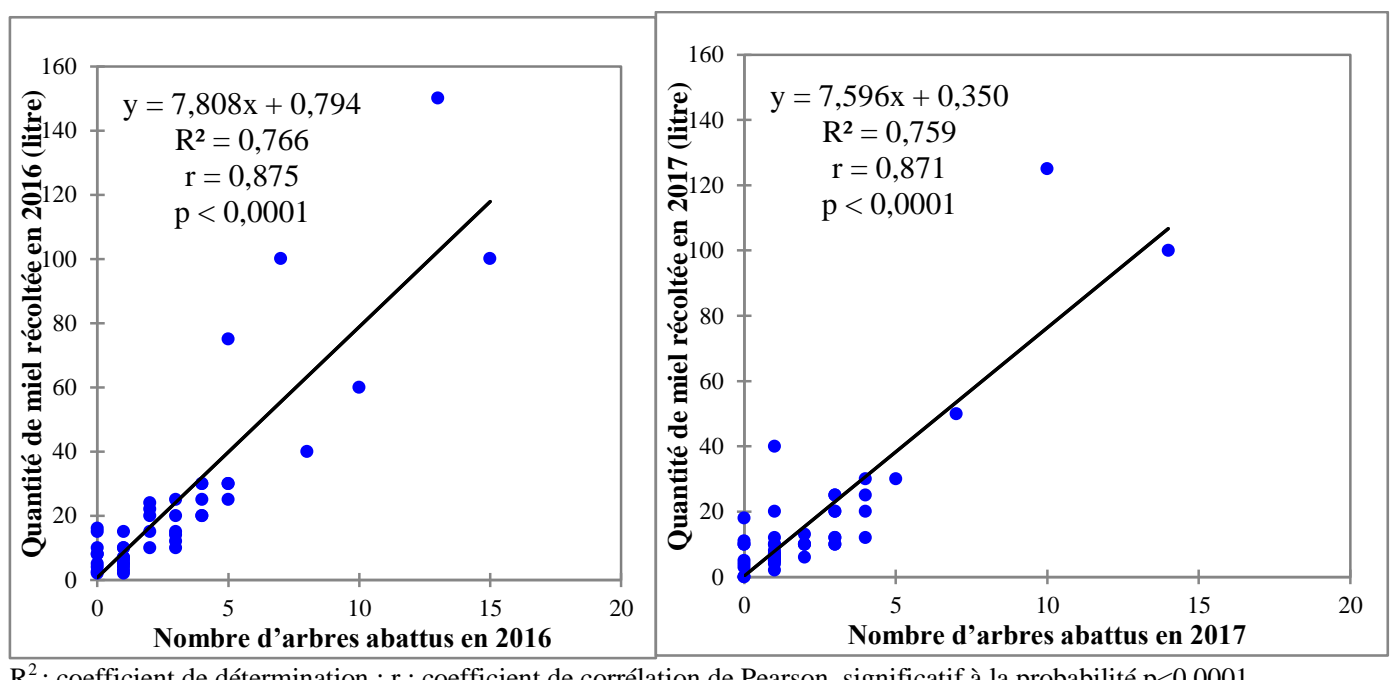

$\mathrm{R}^{2}$ : coefficient de détermination $; \mathrm{r}$ : coefficient de corrélation de Pearson, significatif à la probabilité $\mathrm{p}<0,0001$.

Figure 3: Corrélation entre la quantité de miel récoltée et le nombre d'arbres abattus en 2016 et 2017.

Tableau 3 : Données sur l'apiculture moderne en 2016 et 2017 dans le Département de Katiola.

\begin{tabular}{lll}
\hline Paramètre évalué & $\mathbf{2 0 1 6}$ & $\mathbf{2 0 1 7}$ \\
\hline Nombre moyen de ruches par apiculteur & $13 \pm 11$ & $13 \pm 13$ \\
Récolte totale de miel des apiculteurs (litres) & 1329 & 1202 \\
Récolte moyenne de miel par apiculteur (litres) & $120,82 \pm 90,73^{\mathrm{a}}$ & $109,27 \pm 91,67^{\mathrm{a}}$ \\
Récolte moyenne de miel par ruche (litres) & $9,22 \pm 5,3$ & $8,92 \pm 3,73$ \\
Prix moyen de vente du litre de miel (F CFA) & $1955 \pm 416$ & $1955 \pm 416$ \\
Recette brute moyenne par apiculteurs (F CFA) & $235909 \pm 181543$ & $209727 \pm 191606$ \\
Coût estimatif de l'équipement apicole (F CFA) & - & $154545 \pm 89782$ \\
\hline
\end{tabular}

Les valeurs moyennes affectées de la même lettre ne sont pas significativement différentes au seuil de 5\%.

Tableau 4 : Comparaison de la production moyenne de miel par colonie d'abeilles par l'apiculture et la "chasse au miel".

\begin{tabular}{llll}
\hline Année & $\begin{array}{l}\text { Récolte de miel par ruche } \\
\text { moderne (litres) }\end{array}$ & $\begin{array}{l}\text { Récolte de miel par arbre } \\
\text { (litres) }\end{array}$ & Statistique du test \\
\hline $\mathbf{2 0 1 6}$ & $9,22 \pm 5,3^{\mathrm{a}}$ & $5,92 \pm 7,4^{\mathrm{b}}$ & $\begin{array}{l}x^{2}=7,59 \\
p=0,006\end{array}$ \\
& & $5,8 \pm 7,96^{\mathrm{b}}$ & $\begin{array}{l}\mathrm{F}=16,7 \\
p=0,000\end{array}$ \\
\hline
\end{tabular}

Les valeurs moyennes affectées de la même lettre ne sont pas significativement différentes au seuil de 5\%. 


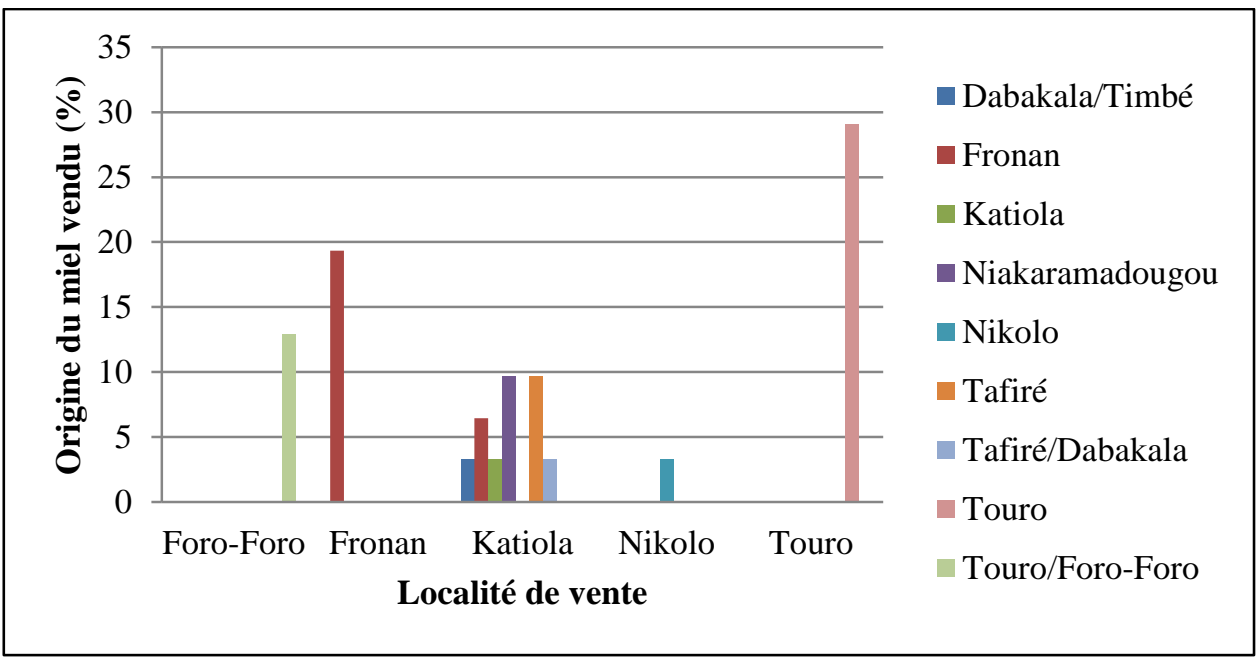

Figure 4: Origine du miel vendu en fonction des localités d'exercice des commerçantes.

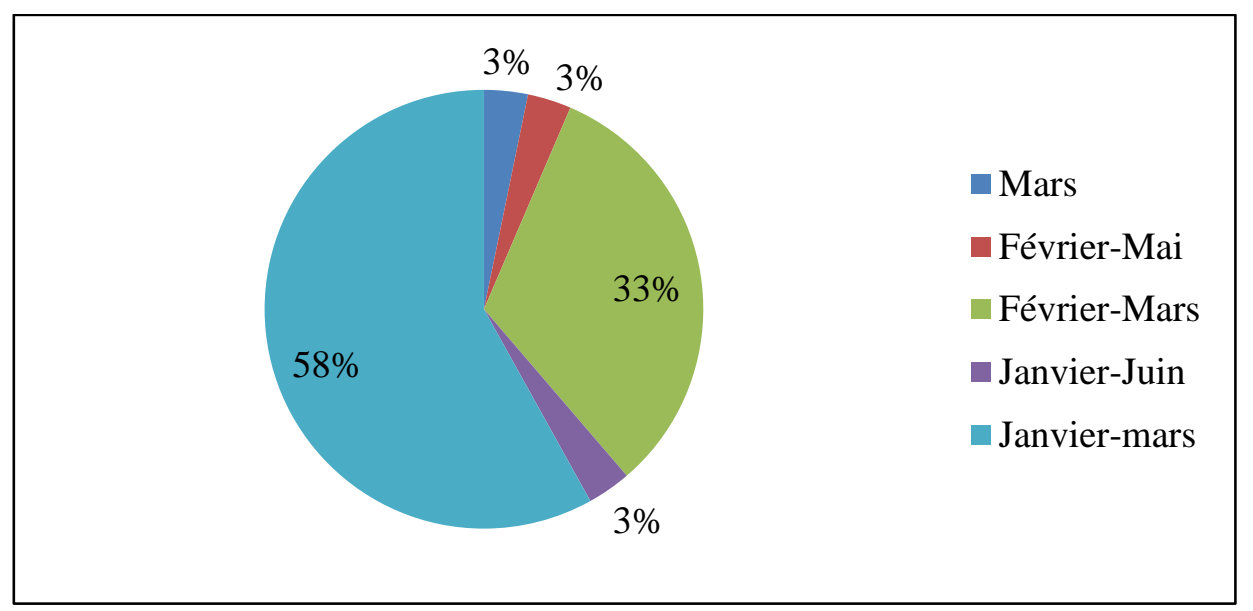

Figure 5: Répartition des commerçantes de miel selon leurs périodes d'approvisionnement.

Tableau 5 : Données sur le commerce du miel dans le Département de Katiola en 2016 et 2017.

\begin{tabular}{lll}
\hline Paramètre évalué & $\mathbf{2 0 1 6}$ & $\mathbf{2 0 1 7}$ \\
\hline $\begin{array}{l}\text { Quantité totale de miel acheté par toutes les commerçantes } \\
\text { (litres) }\end{array}$ & 2535 & 2076 \\
Quantité moyenne de miel acheté par commerçante (litres) & $81,77 \pm 47,33$ & $66,97 \pm 43,44$ \\
$\begin{array}{l}\text { Prix moyen d'achat du litre de miel (F CFA) } \\
\text { Dépense totale pour l'achat du miel par commerçante (F CFA) }\end{array}$ & $1500 \pm 225$ & $1500 \pm 225$ \\
$\begin{array}{l}\text { Dépense totale pour le transport et les emballages par } \\
\text { commerçante (F CFA) }\end{array}$ & $4890 \pm 7815$ & $4130 \pm 7475$ \\
$\begin{array}{l}\text { Prix moyen de vente du litre de miel (F CFA) } \\
\text { Recette moyenne brute par commerçante (F CFA) }\end{array}$ & $2010 \pm 250$ & $2010 \pm 250$ \\
Bénéfice net moyen par commerçante (F CFA) & $180565 \pm 104271$ & $148774 \pm 101110$ \\
\hline
\end{tabular}




\section{DISCUSSION}

Les travaux scientifiques sur la filière miel en Côte d'Ivoire sont rares à notre connaissance. La présente étude a permis d'avoir une meilleure connaissance de la filière dans le Département de Katiola. Comme cela est très souvent le cas en Afrique, les activités autour du miel sont exercées selon le sexe (Betayene, 2008 ; Vestalys et Andrianarivelo-Andriatoavina, 2008). Dans la zone de l'étude, seule la gente masculine pratique la "chasse au miel" et l'élevage des abeilles (apiculture moderne), tandis que les femmes se chargent de la commercialisation du miel. Cela s'expliquerait par le fait que la "chasse au miel" et l'apiculture nécessitent une certaine vigueur et surtout de la réactivité face aux piqûres d'abeilles notamment. Savadogo et al. (2018) ont fait le même constat dans le District de Yamoussoukro au centre de la Côte d'Ivoire. Il en est de même pour Paraiso et al. (2012) au Centre-Nord du Benin et pour Koudegnan et al. (2015) dans la zone écologique IV du Togo. Toutefois, ce constat est contraire à celui fait par plusieurs auteurs (Hussien, 2001; Tsafack et al., 2011; Ahouandjinou et al., 2016) dans des pays de l'Afrique de l'Est et dans certains pays de l'Afrique de l'Ouest et du Centre tels que le Burkina Faso, le Nigeria, le Cameroun où les femmes pratiquent l'apiculture moderne.

S'il faut aller jusqu'à 35 ans d'âge pour observer un apiculteur moderne, il n'en est pas de même pour la chasse au miel. En effet, mêmes des adolescents de 15 ans pratiquent la chasse au miel et ce, depuis près de 2 ans. Ceci pourrait s'expliquer par le fait que les enfants accompagnent toujours les adultes au champ et à la chasse. Ils ont pour rôle essentiel le transport du matériel de chasse (seaux, bidons, torches, etc.). Ainsi, ils en acquièrent de l'expérience dès le bas âge leur permettant de pratiquer seuls la "chasse au miel" quelques années plus tard.

L'âge moyen des apiculteurs modernes (48 ans) plus élevé que celui des autres acteurs de la filière (chasseurs et commerçantes) montre le vieillissement de cette activité et le risque de sa disparition à long terme, en dépit de son importance capitale à plusieurs égards (revenu supplémentaire, biodiversité, pollinisation, etc.). Cette triste réalité serait due au fait que l'apiculture moderne nécessite non seulement un capital initial plus ou moins élevé, mais aussi des connaissances techniques de base. Ce résultat corrobore celui de Yédomonhan et Akoègninou (2009) qui ont rapporté une moyenne d'âge de 50 ans pour les apiculteurs du Centre du Benin, de même que celui de Ahouandjinou et al. (2016) qui ont trouvé 40 ans pour les apiculteurs du Nord-Ouest du Benin.

Depuis des milliers d'années, les populations rurales pratiquent la "chasse au miel" pour plusieurs de leurs besoins (FAO, 2010). Dans le Département de Katiola, plusieurs hommes des zones rurales s'y adonnent chaque année entre janvier et avril. En effet, les quatre premiers mois de l'année constituent la grande période de miellée (production du miel). Ceci s'explique par le fait qu'en zone de savane, la grande floraison des plantes ligneuses qui sont plus pourvoyeuses en nectar (principale matière première du miel), a lieu de décembre à mars (Coulibaly, 2014). C'est donc durant cette période, où les abeilles butinent fortement pour emmagasiner une grande quantité de miel, que se fait la récolte par les "chasseurs de miel".

Bien que la majorité des "chasseurs de miel" (82\%) appartient à un groupe, certains pratiquent seuls cette activité. La chasse solitaire est souvent occasionnée par la présence de ruches naturelles (sauvages) dans des plantations, faisant de leurs propriétaires les détenteurs légitimes de ces ruches qu'ils récoltent chaque année en veillant à sauvegarder les colonies.

Selon les chasseurs, le miel s'obtient de moins en moins ces dernières années qu'il y a 5 ans. Le principal facteur à incriminer pour la raréfaction de plus en plus importante du miel, est certainement la déforestation 
causée non seulement par l'agriculture traditionnelle (Koulibaly et al., 2016) mais aussi par la "chasse au miel" qui induit la destruction quasi systématique des arbres et des colonies d'abeilles. De plus, l'anacarde suscitant de plus en plus d'intérêt chez les paysans, l'espace est exploité majoritairement pour l'installation de plantations d'anacardiers.

La forte corrélation entre la quantité de miel récoltée et le nombre d'arbres abattus met en cause la méthode de récolte employée par les chasseurs. En effet, lorsqu'ils repèrent de jour une colonie sauvage d'abeilles dans le creux d'un arbre, ils viennent de nuit pour récolter le miel. Si la colonie est inaccessible du fait de la morphologie de l'arbre qui ne peut être grimpé, ils l'abattent à coups de hache ou de machette (Yanggen et al., 2010). Il arrive aussi qu'ils abattent souvent des arbres sans y trouver de miel suffisamment mûr. Cela conduit ainsi à la récolte de miel non mûr, donc de mauvaise qualité. Par ailleurs, quand bien même ils trouveraient du miel mûr, ils prélèvent tous les rayons sans distinguer ceux du couvain de ceux du miel mûr.

Malgré l'introduction de l'apiculture moderne dans le Département de Katiola depuis 1973 (Hussien, 2001), cette activité reste très peu pourvoyeuse en miel. En effet, toutes les commerçantes de miel interviewées ne sont fournies que par des "chasseurs de miel". Cette absence de contribution de l'apiculture moderne au miel du marché local pourrait s'expliquer par la rareté des apiculteurs due en partie à la crise militaropolitique de 2002. Aussi, les apiculteurs modernes, mieux organisés (coopérative) et produisant du miel de meilleure qualité, le vendent mieux dans les grandes villes telles que Abidjan et Bouaké. Enfin, la "chasse au miel" reste une activité moins contraignante financièrement, où l'on ne fait que du profit.

Comparativement à la "chasse au miel", l'apiculture moderne permet d'avoir plus de miel par colonie d'abeilles exploitée, malgré le fait que les apiculteurs prennent le soin de toujours laisser des rayons de miel dans les ruches au moment de la récolte pour la pérennité de la colonie. Ceci pourrait s'expliquer par le fait que les ruches modernes sont conçues pour laisser plus d'espace permettant de stocker une grande quantité de miel. En effet, selon Leven et al. (2005), plus une ruche est spacieuse, plus la quantité du miel produite par la colonie en son sein est élevée. L'apiculture moderne, en plus de ses avantages en faveur de la préservation de la biodiversité, garantit une disponibilité constante du miel dans les régions où elle est pratiquée.

Le faible nombre de ruches détenues par apiculteur moderne dans le Département de Katiola (15 ruches), comparativement à celui observé en Centrafrique (40 à 70 ruches) par Mbétid-Bessane (2004) pourrait s'expliquer par le fait qu'en Côte d'Ivoire l'apiculture est considérée comme une activité secondaire. Contrairement à la moyenne de prix pratiquée au Benin, soit 1600 francs CFA (Ahouandjinou et al., 2016), celle observée ici (1955 francs CFA) pourrait s'expliquer par le fait que les apiculteurs enquêtés vendent leur miel à travers la coopérative ou dans les grandes villes où le pouvoir d'achat des consommateurs est plus élevé.

Par ailleurs, nous avons noté que les commerçantes de miel vendent toujours d'autres produits (fruits, légumes, charbon de bois, etc.). Cela s'explique naturellement par la nécessité de diversifier leurs sources de revenus face aux incertitudes de ce commerce imprévisible.

Au regard de la quantité totale de miel vendue par les commerçantes, nous réalisons que le Département de Katiola et, plus généralement la région toute entière, demeure une importante zone à hautes potentialités apicoles. En considérant la correspondance selon laquelle un litre de miel équivaut à 1,4 kilogramme en moyenne (Yahia, 2015), c'est environ 1,5 tonne de miel qui a été vendue par ces commerçantes en 2016 et 1,01 tonne en 2017. 
En Côte d'Ivoire, le miel est considéré comme un remède. Cela justifie sa consommation irrégulière par les populations et donc les difficultés de son écoulement sur le marché local. Néanmoins, le commerce du miel reste une activité rentable où le risque de perte est mineur à cause de la possibilité de sa conservation sur une longue durée.

\section{Conclusion}

Dans le département de Katiola, la majeure partie de la production de miel provient de la "chasse au miel". Cette activité, secondaire dans les zones rurales, est malheureusement à l'origine de la destruction de nombreux arbres et de colonies d'abeilles chaque année. L'apiculture moderne, bien qu'ayant débuté en Côte d'Ivoire par ce département, y reste très peu développée. Les quelques apiculteurs modernes de la région vendent leur miel dans les grandes villes ou à travers leur coopérative pour plus de profits. Toutefois, cette région reste une zone à haute potentialité mellifère qui, en 2016 et 2017, a mis sur le marché 2396 litres et 1985 litres de miel respectivement, quasi-exclusivement commercialisés par des femmes. Il apparaît, à travers le commerce du miel, que l'apiculture est une activité prometteuse. Ainsi, l'orientation des "chasseurs de miel" vers l'apiculture moderne pourrait garantir une production plus importante de miel tout en préservant la biodiversité.

\section{CONFLIT D'INTÉRÊTS}

Les auteurs déclarent qu'ils n'ont aucun conflit d'intérêts.

\section{CONTRIBUTIONS DES AUTEURS}

DO a conçu et supervisé le projet de recherche; DFK a financé et récolté les données sur le terrain ; SC et DFK ont analysé les données et rédigé le manuscrit ; DFK, DO, $\mathrm{SC}$ et KEN ont corrigé et approuvé la version finale du manuscrit.

\section{REFERENCES}

Ahouandjinou TB, Yédomonhan H, Adomou AC, Tossou MG, Akoegninou A. 2016.
Caractéristiques techniques et importance socio-économique de l'apiculture au Nord-Ouest du Bénin : cas de la commune de Cobly. Int. J. Biol. Chem. Sci., 10(3): 1350-1369. DOI : https://www.ajol.info/index.php/ijbcs/art icle/view/149070/138572

Betayene D. 2008. Débuter en apiculture. Centre pour l'Environnement et le Développement (CED), Cameroun, Yaoundé, 44 p. DOI : https://www.apiservices.biz/documents/a rticles-

fr/manuel_formation_apicole_cameroun. pdf

Coulibaly S. 2014. Potentialités de production mellifère de la flore de transition forêtsavane, en zone Guinéenne et caractérisations pollinique et physicochimique de quelques miels de la Côte d'Ivoire (Afrique de l'Ouest). Thèse de Doctorat, Université Félix HouphouëtBoigny, Abidjan, Côte d'Ivoire, p. 194.

Dieye PN, Faye A, Seydi M, Cissé SA. 2002. Production laitière périurbaine et amélioration des revenus des petits producteurs en milieu rural au Sénégal. Cahiers Agriculteurs, 11: 251-257. DOI :

http://revues.cirad.fr/index.php/cahiersagricultures/article/view/30351

FAO. 2010. Le rôle des abeilles dans le développement rural, manuel sur la récolte, la transformation et la commercialisation des produits et services dérivés des abeilles p. 238. DOI :

http://www.fao.org/docrep/013/i0842f/i0 842f00.pdf

Hussein MH. 2001. L'apiculture en Afrique. Apiacta., 1: 34-48. DOI: http://www.apimondiafoundation.org/pdf Kouakou CK, Akanvou L, Konan YA, Mahyao A. 2010. Stratégies paysannes de maintien et de gestion de la biodiversité du maïs (Zea mays L.) dans le Département de Katiola, Côte d'Ivoire. Journal of Applied Biosciences, 33: 2100-2109. DOI: 
http://www.m.elewa.

org/JABS/2010/33/9.pdf

Koudegnan C, Nenonene A, Guelly K, Edorh

T. 2015. L'apiculture dans la lutte contre les changements climatiques dans la zone écologique IV du Togo. Afrique Science, 11(6): 45-59. DOI : http://www.afriquescience.info/documen t.php?id=5480

Koulibaly A, Goetze D, Traoré D, Porembski S. 2006. Protected versus exploited savanna: characteristics of the Sudanian vegetation in Ivory Coast. Candollea, 61(2): 425-452. DOI: https://www.villege.ch/cjb/publications/cando612/C612_4 25-452_abstr.pdf

Koulibaly A, Akédrin N, Diomandé M, Konaté I, Traoré D, Bill R, Kouadio YJ. 2016. Conséquences de la culture de l'anacardier (Anacardium occidentale L.) sur les caractéristiques de la végétation dans la région du Parc National de la Comoé (Côte d'Ivoire). IJIAS, 17(4): 1416-1426. DOI : http://www.ijias.issr-

journals.org/fr/authid.php?id=7302

Lebel F, Debailleul G, Samba S, Olivier A. 2002. Contribution des produits forestiers non- ligneux à l'économie des ménages de la région de Thiès, au Sénégal. In: Acte du $2^{\text {ème }}$ Atelier Régional sur les Aspects socioéconomiques de l'agroforesterie au Sahel, 4-6 mars 2002, Bamako, Mali, 19.

Leven L, Boot W, Mutsaers M, Segeren P, Velthuis H. 2005. L'apiculture dans les zones tropicales. Agrodok, 32: 94. DOI: https://www.scribd.com/document/3364 63871/AD32F-L-Apiculture-Dans-LesZones-Tropicales

Mbetid-Bessane E. 2004. Apiculture, source de diversification des revenues des petits agriculteurs: cas du bassin cotonnier en Centrafrique. Tropicultura, 22(3): 156158.

DOI : http://pdfmyurl.com/?url=http://www.slir e.net/document/1984?pdfmyurl=fr\&versi on=pdf\&-s=A4\&--

filename=slire_document_1984_fr_2018 -10-01.pdf

Paraiso A, Olodo GP, Tokoudagba S, Auteu R, Yegbemey RN, Sanni A. 2012. Déterminants et contraintes de la production du miel dans le Nord-Ouest du Bénin : Cas des communes de Natitingou et de Tanguiéta. Journal de la Recherche Scientifique de l'Université de Lomé, 14(1): 69-82. DOI : https://www.ajol.info/index.php/jrsul/arti cle/view/86759

Savadogo S, Assi Kaudjhis C, N'Guessan K. 2018. Note sur la place de l'apiculture dans la société Baoulé en Côte d'Ivoire : cas de deux villages du District de Yamoussoukro. Geo-Eco-Trop, 42(1): 199-206.

DOI: http://www.geoecotrop.be/uploads/publi cations/pub_421_14.pdf

Tamboura H, Kaboré H, Yaméogo SM. 1998. Ethnomédecine vétérinaire et pharmacopée Traditionnelle dans le plateau central du Burkina Faso : cas de la province du Passoré. Biotechnol. Agron. Soc. Environ., 2(3): 81-191. DOI :

http://www.pressesagro.be/ojs/index.php /base/article/viewFile/57/57

Tousso AGE. 2005. Analyse socioéconomique du système de commercialisation des amendes et du beurre de karité (Vitellaria paradoxa) dans les communes de Kandi et Gogounou. Thèse d'Ingénieur Agronome, Faculté des Sciences Agronomique, Université d'Abomey Calavi, Benin, p. 65.

Tsafack MAS, Muluh AG, Kamajou F, Verina I, Vabi BM. 2011. Etude comparative de la rentabilité de deux types d'apiculture au Nord-Ouest Cameroun. Tropicultura, 29(1):

3-7.

DOI : 
http://www.tropicultura.org/text/v29n1/3 .pdf

UE. 2014. Directive 2014/63/UE du Parlement Européen et du Conseil du 15 mai 2014 modifiant la directive 2001/110/CE du Conseil relative au miel. Journal officiel de l'Union Européenne. DOI : https://eurlex.europa.eu/legalcontent/FR/TXT/HTML/?uri=OJ:JOL_2 014_164_R_0001\&from=FR

Vestalys $\mathrm{H}$, Andrianarivelo-Andriatoavina MS. 2008. Analyse de la filière apiculture dans les régions Analamanga et haute Matsiatra. Etude De Cas Programme Pays Madagascar Direction: FIDA et FAO. PROSPERER, $43 \mathrm{p}$.
Yahia MS. 2015. Analyses physico-chimique du miel de quelques miels de la wilaya : Ain Defla, Djendel, Bathia, Bourached et Miliana. Mémoire de Master, Université Djilait Bounaama, Algerie, p. 85.

Yanggen D, Angu K, Tchamou N. 2010. Landscape-Scale Conservation in the Congo Basin: Lessons Learned from the Central African Regional Program for the Environment (CARPE). Gland, Switzerland: IUCN, p. 262.

Yédomonhan H, Akoègninou A. 2009. La production du miel à Manigri (Commune de Bassila) au Bénin: enjeu et importance socio-économique. Int. J. Biol. Chem. Sci., 3(1): 125-134. DOI : https://www.ajol.info/index.php/ijbcs/arti cle/view/42743 